\title{
LAS CONSTRUCCIONES ESCOLARES EN ESPAÑA EN LOS AÑOS VEINTE Y TREINTA DEL SIGLO PASADO (LA DICTADURA PRIMORRIVERISTA Y LA SEGUNDA REPÚBLICA)
}

\author{
School buildings in Spain in the twenties and thirties \\ of the past century (Primo de Rivera Dictatorship \\ and the Second Republic)
}

\author{
Francisco Javier Rodríguez Méndez ${ }^{\alpha}$, \\ María del Pilar García Salmerón ${ }^{\beta}$ \\ y Antonio Viñao Fragor
}

\section{INTRODUCCIÓN}

Tras una larga fase de manipulación, denigración, desmemoria u olvido por la historiografía educativa de lo que había sido o intentado ser la educación durante la Segunda República, en la segunda mitad de los años setenta comenzaron a aparecer los primeros libros dedicados monográficamente al tema a cargo de Mariano Pérez Galán (1975), Antonio Molero Pintado (1977) y Mercedes Samaniego Boneu (1977), si bien el primero dejaba fuera de su análisis los años de la guerra civil y los otros dos se limitaban a los ministerios de Marcelino Domingo y Fernando de

\footnotetext{
${ }^{\alpha}$ Escuela Politécnica Superior de Zamora. Universidad de Salamanca. Avda. Cardenal Cisneros, 34 (Campus Viriato) 49029 Zamora. España. rodmen@usal.es

B Calle Hermanos Becerril 34, 4 A. 16004 Cuenca. España. pgarciasalmeron@gmail.com

$\gamma$ Facultad de Educación. Universidad de Murcia. Campus universitario de Espinardo. 30100 Murcia.España. avinao@um.es
}

Cómo citar este artículo: Rodríguez Méndez, Francisco Javier, María del Pilar García Salmerón y Antonio Viñao Frago. " Las construcciones escolares en España en los años veinte y treinta del siglo pasado (La dictadura primorriverista y la Segunda República)». Historia y Memoria de la Educación 11 (2020): 649-673 
los Ríos durante el bienio azañista de 1931-1933. ${ }^{1}$ Redactados dichos estudios en los años del tardofranquismo y/o la transición hacia un nuevo régimen político, a ellos habría que añadir la traducción en 1978 del libro de Ramón Safón sobre la educación en la zona republicana durante la guerra civil, así como, ya en la década de los ochenta, los de Claudio Lozano Seijas (1980), Fernando Millán (1983) sobre la «revolución laica» y Juan Manuel Fernández Soria (1984) sobre la educación durante la guerra civil en ambas zonas. ${ }^{2}$ De un modo u otro, todos ellos fueron escritos y publicados en el contexto de lo que podríamos llamar un primer intento de recuperación historiográfica de este período de nuestra historia.

Esta relación, sin pretensiones exhaustivas, ${ }^{3}$ de estudios de carácter general y diferentes delimitaciones temporales, comenzó a verse acompañada, en una segunda fase, por una serie de trabajos de ámbito local, comarcal, provincial o regional sobre la educación en la Segunda República, o sobre aspectos determinados de la misma, desde la década de los ochenta y, sobre todo, durante los años noventa del siglo XX y los primeros años del siglo XXI, de tal modo que puede afirmarse que, contra cierta lógica, en este campo los estudios de índole general, que abarcaban todo el país, precedieron en el tiempo a aquellos territorial o temáticamente más limitados. ${ }^{4}$ De ahí que estos trabajos posteriores sirvan para comprobar hasta qué punto fueron o no efectivas y se aplicaron algunas de las

\footnotetext{
1 Mariano Pérez Galán, La enseñanza en la Segunda República española (Edicusa: Madrid 1975), Antonio Molero Pintado, La reforma educativa de la Segunda República española. Primer bienio (Santillana: Madrid 1977), y Mercedes Samaniego Boneu, La política educativa de la II República durante el bienio azañista (C.S.I.C.: Madrid 1977).

${ }^{2}$ Ramón Safón, La educación en la España revolucionaria (Las Ediciones de La Piqueta: Madrid. 1978), Claudio Lozano, La educación republicana, 1931-1939 (Universidad de Barcelona: Barcelona 1980), Fernando Millán, La revolución laica: de la Institución Libre de Enseñanza a la escuela de la República (Fernando Torres: Valencia 1983), y Juan Manuel Fernández Soria, Educación y cultura en la guerra civil (España 1936-1939) (Nau Llibres: Valencia 1984).

${ }_{3}^{3}$ Como es obvio, durante los años setenta y ochenta aparecerían artículos, asimismo generales sobre el tema, y se le dedicarían algunos capítulos en libros más amplios sobre la Segunda República. Valgan, a título de ejemplo, los de Matilde Vázquez, «La reforma educativa en la zona republicana durante la guerra civil», Revista de Educación 240 (1975): 60-72, Miguel Ángel González Muñiz, Problemas de la Segunda República (Ediciones Júcar; Madrid 1974), 327-407, y Carlos Alba Tercedor, «La educación en la II República: un intento de socialización política», en Estudios sobre la Segunda República española, ed. Manuel Ramírez (Tecnos; Madrid 1975), 47-75.

${ }^{4}$ Un análisis comparativo de dichos estudios, hasta un total de 33, que la misma autora declara no exhaustivo, es el de María del Pilar García Salmerón, «La política y las realizaciones educativas de la segunda República, a la luz de los estudios locales», Sarmiento. Anuario galego de historia da educación 8 (2004): 103-125.
} 
medidas, políticas y disposiciones generales sobre la educación en la Segunda República. Por ejemplo, las relativas a la creación y construcción de escuelas, la retirada de los símbolos religiosos de las escuelas, y otras medidas de signo laicista, o la Ley de Confesiones y Congregaciones Religiosas de 1933, por citar algunos de los temas más controvertidos.

La cuestión relativa a la creación y construcción de escuelas fue objeto de debate ya desde las primeras publicaciones. En especial, al cuantificar el «esfuerzo» escolarizador republicano, cualquier lector podía apreciar diferencias en las cifras que se ofrecían en los estudios mencionados. Los cálculos sobre el número de docentes del magisterio primario o de escuelas construidas o creadas desde 1931 a 1936 diferían según la perspectiva o criterios empleados por quien los efectuaba.

Dos tipos de investigaciones relacionadas entre sí —-sobre la evolución general de las construcciones escolares en la España del primer tercio del siglo XX, en un caso, y sobre la determinación comparativa de dicho «esfuerzo» constructor entre la dictadura primorriverista (19231930) y la Segunda República (1931-1936), en otro-, junto con la celebración de diversas exposiciones y las publicaciones aparecidas, en torno al 2006, con motivo del 75 aniversario de la proclamación de la Segunda República, replantearon dicho debate desde una nueva perspectiva: la de las continuidades y los cambios —si los hubo y en qué sentido- entre la política de construcciones escolares llevada a cabo durante la dictadura de Primo de Rivera y la Segunda República.

Un artículo, publicado en 2013, de María del Pilar García Salmerón, redactado tras una investigación minuciosa, reabrió la polémica, nunca cerrada, con el fin de rebatir, en este punto, las «inexactitudes descomunales» que, a su juicio, se vertían en el catálogo de la exposición La escuela de la II República organizada en 2007 por la Fundación de Investigaciones Educativas y Sindicales (FIES) de la Federación de Enseñanza de Comisiones Obreras en relación, entre otros aspectos, el «plan masivo de construcciones escolares» que, según dicho catálogo, se llevó a cabo en la Segunda República y las cifras ofrecidas en el mismo sobre el incremento en el número de maestros. ${ }^{5}$ Según la síntesis efectuada por la autora en el resumen inicial,

\footnotetext{
${ }^{5}$ María del Pilar García Salmerón, «Radiografía de las construcciones escolares públicas en España (1920-1937): la imagen distorsionada de la II República», Aportes 28, no 83 (2013): 21-52 (cita en p. 23).
} 
La situación generalizada de penuria de los locales-escuela en España comenzó a cambiar a partir de la década de los veinte. La mejora y expansión del parque público de edificios escolares se vio favorecida por factores como la implicación del Estado y la habilitación de presupuestos extraordinarios. El tiempo y forma en la expansión de la red escolar pública fue marcada por los ayuntamientos de todo signo político. El cálculo global, referido al número de escuelas de nueva planta alzadas durante esta etapa con el auxilio económico estatal demuestra que, durante los años que median entre 1922 y 1931 se levantaron en España más escuelas que durante la II República. Queda desmontado el tópico que atribuye al advenimiento republicano el mérito del mayor incremento en las infraestructuras escolares de la historia contemporánea. ${ }^{6}$

Sus conclusiones, tras un cómputo minucioso de las escuelas/aulas construidas en dichos años, eran tajantes:

[...] en los años veinte se alzaron en España en torno a mil doscientos edificios escolares, en los que tendrían cabida más de cuatro mil aulas. Durante la II República se concluyeron las obras de unos setecientos edificios escolares, con una cifra cercana a las tres mil aulas.

Por tanto, el discurso tan extendido y aceptado que encumbra a la República como el régimen político pionero y responsable de una drástica mejora en las infraestructuras escolares debe cuestionarse seriamente. ${ }^{7}$

La reciente publicación por García Salmerón de la monumental por su extensión- y documentada Radiografía de las construcciones escolares públicas en España, 1922-1937 (Madrid: Ministerio de Educación y Formación Profesional, 2018, 777 páginas. ISBN 978-84-369-5883-6), prologada por Gabriela Ossenbach Sauter, fue el origen de la recepción en Historia y Memoria de la Educación de un comentario-reseña redactado por otro de los mejores conocedores de las construcciones

\footnotetext{
6 García Salmerón, «Radiografía de las construcciones escolares públicas en España (1920-1937)», 21.

7 García Salmerón, «Radiografía de las construcciones escolares públicas en España (1920-1937)», 37-38.
} 
escolares en la España del primer tercio del siglo XX, Francisco Javier Rodríguez Méndez, autor asimismo de algún estudio sobre la debatida cuestión del cómputo de las escuelas construidas y creadas durante la Segunda República. Su lectura nos hizo pensar en la posibilidad de que García Salmerón, a la vista de dicho comentario-reseña, ofreciera su punto de vista sobre lo que en ella se decía, abriendo así la sección de debates de la revista. Una sección que, en este caso, se cerraría con la aportación, por parte de Antonio Viñao, como estudioso asimismo de las construcciones escolares y de la escolarización en los años mencionados, de unas reflexiones finales más de índole metodológica que conclusivas.

Dicho esto, a modo de presentación, damos paso a la cuestión debatida siguiendo el orden de intervenciones indicado.

\section{FRANCISCO JAVIER RODRÍGUEZ MÉNDEZ}

María del Pilar García Salmerón es Licenciada en Filosofía y Ciencias de la Educación por la UNED y Doctora en Filosofía y Ciencias de la Educación, también por la UNED. Compatibiliza su vocación docente, ejercida en el CEIP «Fuente del Oro» de Cuenca, con la investigación en el campo de las políticas y realizaciones educativas de la segunda República. Se trata de una línea de investigación —explica Gabriela Ossenbach, su directora de tesis y autora del prólogo- en la que la autora lleva trabajando largos años, a lo largo de los cuales ha ido anticipando parte del libro ahora publicado. Destaca en primer lugar su Tesis doctoral, ${ }^{8}$ defendida en 1999 y publicada algo después con el título Educación y República en Cuenca, 1931-1939. ${ }^{9}$ Los artículos titulados La política y las realizaciones educativas de la segunda República, a la luz de los estudios locales ${ }^{10}$ —de 2004- y Radiografía de las construcciones escolares públicas en España (1920-1937): la imagen distorsionada de la

\footnotetext{
\& María del Pilar García Salmerón, La enseñanza en Cuenca durante la II República y la Guerra Civil Española. Tesis doctoral (Madrid: Universidad Nacional de Educación a Distancia, 1999).

9 María del Pilar García Salmerón, Educación y República en Cuenca, 1931-1939 (Cuenca: Diputación Provincial de Cuenca, 2003).

${ }_{10}$ García Salmerón, «La política y las realizaciones educativas de la segunda República a la luz de los estudios locales», Sarmiento. Anuario galego de historia da educación 8 (2004): 103-125.
} 
II República ${ }^{11}$ — de 2013 - constituyen la base en que se sustenta la publicación que ahora se nos brinda.

No son pocas las similitudes entre el perfil de la autora y el de quien esto escribe. Además del año de nacimiento, compartimos la pasión por el mismo tema de investigación —ella desde su formación en Educación y yo desde la mía como arquitecto- y también, quizás, nuestra condición de outsiders por el hecho de ejercer la docencia en aulas más o menos alejadas de nuestro principal interés. No nos conocemos personalmente, pero anduvimos cerca de ello cuando en 2014 participé en las VI Jornadas de la SEPHE con la contribución titulada Pero, ¿cuántas escuelas construyó realmente la República?.12 Algún tiempo después, Gabriela Ossenbach me propuso colaborar con Pilar García Salmerón, dada la coincidencia entre nuestros intereses. En aquel momento, tras darle muchas vueltas, decliné el ofrecimiento, y pienso que lo hice, en parte, por lealtad debida en el contexto de una contienda académica. Ahora, pasados cinco años, se me remite por la misma vía el libro de Pilar García para que redacte una reseña. A la vista de la alta calidad del resultado, debiera lamentarme de aquella negativa, que es lo que quizás se busca, pero... así son las cosas. Y, dicho lo cual, entro en materia.

El libro está estructurado en tres capítulos, las conclusiones y un apéndice documental que ocupa algo más de la mitad de las 777 páginas de la publicación. El primero de los capítulos estudia con gran profundidad el marco legal, económico y social de las construcciones escolares públicas durante el período 1922-1937. Frente a la opinión generalizada hasta ahora de que fue principalmente a partir del advenimiento de la II República cuando las construcciones escolares cobraron especial auge, la autora pone en valor la labor desempeñada en este campo durante el Directorio Militar, así como el importante papel desempeñado por las Cajas de Ahorro y el Instituto Nacional de Previsión. Y lo hace basándose no en opiniones, sino en los resultados reflejados en las tablas del anexo documental, que, resumidos en el cuadro de la página 307, no dejan lugar a dudas para la autora: «En los años veinte se alzaron en

\footnotetext{
${ }^{11}$ García Salmerón, «Radiografía de las construcciones escolares públicas en España (1920-1937): la imagen distorsionada de la II República», Aportes 28, nº 83 (2013): 21-52.

12 Francisco Javier Rodríguez Méndez, «Pero, ¿cuántas escuelas construyó realmente la República? Parte primera: 1936-1939», en Actas de las VI Jornadas Científicas de la Sociedad Española para el Estudio del Patrimonio Histórico Educativo (Madrid, 2014), 567-575.
} 
España en torno a mil doscientos edificios escolares, en los que tendrían cabida más de cuatro mil aulas. Durante la II República se concluyeron las obras de unos setecientos edificios escolares, en los que se instalarían unas tres mil aulas».

El progresivo mayor protagonismo de los Municipios frente al Estado en la promoción de las construcciones escolares queda patente de forma palmaria en el cuadro 2.5 de la página 144, cuyas cifras podrían resumirse así: mientras que en el período 1922-1931 los municipios promovieron un total de 1.968 aulas frente a las 2.018 del Estado (lo que implica un $49 \%$ de iniciativa municipal y un 51\% de la estatal), en el período 1931-1937 las tornas cambiaron abrumadoramente a favor de los primeros: 6.957 aulas de los municipios frente a las 2.751 del Estado (lo que implica un $72 \%$ de iniciativa municipal y un $28 \%$ de la estatal). Ahora bien, en este segundo período 1931-1937 las realizaciones no fueron a la par del crecimiento en la asunción de responsabilidades por los Municipios, y así se desprende del hecho de que, mientras que los consistorios promovieron doble cantidad de aulas que el Estado, el cómputo final de aulas terminadas fue similar en ambas modalidades. «Esto parece indicar — concluye la autora - una mayor efectividad en la ejecución de obras tuteladas por el Estado, con la supervisión directa de la Oficina Técnica para la Construcción de Escuelas, frente a las obras controladas por las corporaciones locales» (p. 144).

A la vista de la desigual distribución geográfica de las inversiones edilicias que se desprende de las tablas del anexo II, nos parece muy interesante la reflexión planteada por la autora, quien, ante la flagrante disparidad de las «Coordenadas espacio-temporales de los nuevos edificios», se pregunta si la Dirección General de Primera Enseñanza llegó a implementar algún tipo de medida para paliar las carencias de las regiones más pobres. La conclusión que obtiene, tras un análisis riguroso de los listados por provincias e índices de analfabetismo, es que «no existió una planificación inspirada [...] ni se buscó compensar los desajustes interterritoriales» (p.150).

El segundo capítulo se dedica a analizar cuantitativa y cualitativamente las construcciones escolares públicas entre 1922 y 1937. Y lo hace con una profundidad y exhaustividad como no se había hecho hasta ahora. Todo lo expuesto - en unión de los listados y tablas de los Anexos I 
y II- posee un incalculable valor y servirá de base en los ulteriores estudios de detalle. Se trata de un desarrollo de un artículo de 2013 que compartía título con el actual libro, si bien se subtitulaba de manera diferente (La imagen distorsionada de la República) pero quizás más expresiva respecto a las conclusiones del estudio. A este último estudio se refiere el profesor Antonio Viñao en un reciente artículo, ${ }^{13}$ y a él dedica un extenso epígrafe del mismo ( La polémica sobre la política de construcciones escolares»). Viñao Frago, que no objeta las conclusiones generales de García Salmeron a las que antes nos hemos referido, se posiciona en el debate del recuento de las escuelas, y no tanto para ofrecer sus propias cifras, sino para «señalar algunas cuestiones metodológicas» que «complementan» los postulados de la autora y que podrían ser útiles en futuros inventarios. A dicho artículo remitimos para una mayor profundización en esta materia.

El que esto escribe, por su parte, dio a conocer en 2014 un texto de parecida temática y similares conclusiones, en el que involuntariamente pasamos por alto el artículo de Pilar García de 2013. Se trató, a mi juicio, de una de esas confluencias que se dan en diversos momentos y campos de la ciencia cuando un tema está lo suficientemente maduro. ${ }^{14}$ Estudiosos de una misma materia, a ambos nos llamaron poderosamente la atención la disparidad y exageración de los datos relativos a construcciones escolares durante el período republicano. Por esa razón, y tras acotar en parecidos términos el hecho constructivo y sus agentes, nos volcamos en acometer en primer lugar lo que estaba más a mano: un vaciado íntegro de la Gaceta de Madrid. Ahora bien, mientras que Pilar García llevó a término la prospección y además la completó con otras provenientes de diversos archivos, la nuestra se halla inacabada. Además, y como expresaba el título completo de aquella contribución, ${ }^{15} \mathrm{se}$

\footnotetext{
13 Antonio Viñao Frago, «La "educación republicana" y algunos otros textos de Claudio Lozano», en La historia de la educación entre Europa y América: estudios en honor del profesor Claudio Lozano Seijas, dirs. Olegario Negrín Fajardo, Javier Vergara Ciordia y Conrado Vilanou Torrano (Madrid: Editorial Dykinson, 2018), 103-114.

14 Me consta, al menos, otro recuento efectuado por el colectivo Cazarabet para las provincias aragonesas: El esfuerzo de la República en la construcción de nuevas escuelas en Aragón (1931-1937), a través de la Gazeta [consulta: 16-04-2016]. Disponible en: http://www.cazarabet.com/idea/escuelas/ escuelasrepublica.htm

${ }_{15}$ Rodríguez Méndez, «Pero, ¿cuántas escuelas construyó realmente la República? Parte primera: 1936-1939».
} 
comenzó desde las postrimerías de la República —1939—, llegando con el recuento en aquella ocasión hasta 1936, y a fecha de hoy hasta 1933. A pesar de carecer aún de los datos correspondientes a los años 1931 y 1932, creo que sería interesante comparar someramente los resultados obtenidos hasta la fecha por un servidor con los publicados por Pilar García, y así obtener algunas conclusiones sobre la metodología y los criterios adoptados en cada recuento.

Aunque Pilar García hable siempre de escuelas «proyectadas» para referirse a las aprobadas oficialmente (p. 308), nosotros preferimos hablar de escuelas "programadas», y así lo hemos hecho tanto en el artículo de 2014 como en la tabla comparativa que se adjunta. No son pocos los casos de escuelas proyectadas (es decir, que contaban ya con proyecto arquitectónico supervisado por la Oficina Técnica) cuyo procedimiento no llegó siquiera a iniciarse, a la espera del solar y/o la aportación municipal que nunca se hicieron efectivos, o por el motivo que fuere. Casos estos que, naturalmente, no han de figurar en los listados.

\begin{tabular}{|c|c|c|c|c|c|c|c|c|c|c|c|c|}
\hline \multirow[t]{2}{*}{ AÑ̃ } & \multicolumn{2}{|c|}{$\begin{array}{c}\text { Aulas de } \\
\text { unitarias } \\
\text { programadas }\end{array}$} & \multicolumn{2}{|c|}{$\begin{array}{c}\text { Aulas de } \\
\text { unitarias } \\
\text { Construidas }\end{array}$} & \multicolumn{2}{|c|}{$\begin{array}{c}\text { Aulas de } \\
\text { graduadas } \\
\text { Programadas }\end{array}$} & \multicolumn{2}{|c|}{$\begin{array}{c}\text { Aulas de } \\
\text { graduadas } \\
\text { construidas }\end{array}$} & \multicolumn{2}{|c|}{$\begin{array}{c}\text { TOTAL } \\
\text { programadas }\end{array}$} & \multicolumn{2}{|c|}{$\begin{array}{c}\text { TOTAL } \\
\text { construidas }\end{array}$} \\
\hline & MPGS & FJRM & MPGS & FJRM & MPGS & FJRM & MPGS & FJRM & MPGS & FJRM & MPGS & FJRM \\
\hline 1931 & 61 & ¿? & 40 & $\dot{i} ?$ & 508 & ¿? & 394 & ¿? & 569 & ¿? & 434 & $\dot{c}$ ? \\
\hline 1932 & 212 & $\dot{i} ?$ & 131 & $\dot{i} ?$ & 518 & ¿? & 291 & ¿? & 730 & ¿? & 422 & ¿? \\
\hline 1933 & 687 & 485 & 383 & 133 & 1.344 & 1.313 & 692 & 555 & 2.031 & 1.798 & 1.075 & 688 \\
\hline 1934 & 579 & 552 & 248 & 237 & 1.491 & 1.674 & 513 & 537 & 2.070 & 2.226 & 761 & 774 \\
\hline 1935 & 640 & 661 & 81 & 153 & 1.212 & 1.829 & 151 & 330 & 1.852 & 2.490 & 232 & 483 \\
\hline 1936 & 463 & 814 & 3 & 401 & 994 & 2.445 & 0 & 1.296 & 1.457 & 3.259 & 3 & 1.697 \\
\hline 1937 & 345 & 50 & 2 & 91 & 654 & 180 & 0 & 310 & 999 & 230 & 2 & 401 \\
\hline 1938 & - & 7 & - & 39 & - & 41 & - & 115 & - & 48 & - & 154 \\
\hline Total & 2.987 & 2.569 & 888 & 1.054 & 6.721 & 7.482 & 2.041 & 3.143 & 9.708 & 10.051 & 2.929 & 4.197 \\
\hline
\end{tabular}

Tabla elaborada por el autor de la reseña tomando como base las tablas de las páginas 484-503 del libro de Pilar García Salmerón y sus propios datos.

La tabla anterior se refiere únicamente a la actividad correspondiente al período de vigencia de la II República Española, pues del periodo 
1922-1931 solamente aporta datos la autora del libro. En dicha tabla se especifican, según cada investigador, ${ }^{16}$ las cifras de aulas "programadas» o aulas "construidas», ya se trate de escuelas unitarias, graduadas o de la suma de ambas. Teniendo en cuenta que faltan en un caso los resultados correspondientes a 1931 y 1932, se observa una considerable disparidad entre las cifras totales de aulas programadas -9.708 (MPGS) frente a 10.051 (FJRM) — y más entre las aulas construidas - 2.929 (MPGS) frente a 4.197 (FJRM)—, discrepancias que podrían deberse, más que a errores en el recuento, a los diferentes criterios empleados en su obtención.

Habida cuenta de la carencia antes mencionada, podría pensarse que se da una cierta coherencia entre las cifras de aulas de unitarias programadas - 2.987 (MPGS) y 2.569 (FJRM) - , por implicar un menor recuento en un menor plazo. En cambio, entre las correspondientes a las aulas de graduadas programadas —6.721 (MPGS) y 7.482 (FJRM)— la incoherencia es total, pues se programan más escuelas en un menor plazo. Se intuye que la causa de ello radica en el empleo de diferentes unidades de medida en el caso de las escuelas graduadas. De la lectura de su libro, se desprende que para la profesora García Salmerón computan únicamente aquellas dependencias del edificio escolar destinadas a albergar la actividad docente convencional, es decir, solamente las clases y no las dependencias complementarias tales como biblioteca, aula de trabajos manuales, inspección médica u otras. Es un criterio que establece una relación directa entre inversión económica y escolarización y que coincide con el empleado por el Instituto Nacional de Previsión en sus estadísticas, pero que - a nuestro juicio- no hace justicia al esfuerzo del Ministerio de Instrucción Pública y de los municipios: no es lo mismo una escuela graduada que cuenta con seis clases y nada más, que otra del mismo número que además tiene dependencias complementarias. En nuestra contribución de 2014 decíamos que debían contabilizarse no solamente las salas de clase, sino también otros locales complementarios — biblioteca, cantina escolar, departamento de duchas, museo, Inspección médico-escolar, salas de trabajos manuales, casa del conserje, etc.- y nos basábamos para ello en idéntico criterio al empleado por el Ministerio de Instrucción Pública, tanto en la adjudicación de las subvenciones a los municipios, como en la realización de sus

${ }^{16}$ MPGS: María Pilar García Salmerón. FJRM: Francisco Javier Rodríguez Méndez. 
propios recuentos. A nuestro parecer, tanto unos resultados como otros —obtenidos según uno u otro criterio- revisten interés y son aplicables según sea el segundo término con el que se desee comparar.

Mientras que la programación de una escuela es fácilmente adjudicable al sistema vigente en el momento de la correspondiente publicación en la Gaceta (ese ha sido, al menos, nuestro criterio), no ocurre así en el caso de la construcción. Para García Salmerón las construcciones contabilizadas cada año corresponden únicamente a aquellos edificios que habiendo sido aprobados ese mismo año llegaron a término, sea cual sea la fecha: «[...] los edificios contabilizados como terminados en un ejercicio representan los proyectos aprobados durante esa anualidad y que en años sucesivos, cuando se remataron las obras, llegaron a ser realidad. No deben interpretarse como edificios terminados en ese ejercicio. Normalmente pasaban más de un año o dos desde la aprobación del proyecto hasta que se concluían las obras.» (p. 139). Lo que no se comprende es que, en aplicación de este criterio y para el año 1937, la profesora García Salmerón contabilice 999 aulas programadas y solamente 2 construidas. ¿Debe interpretarse que solo 2 aulas llegaron a terminarse y que 997 fueron revocadas en la posguerra? De haber sido así, estaríamos ante un caso digno de estudio.

Rodríguez Méndez coincide en apreciar el abultado desfase entre programación y construcción: «Se tiene constancia de construcciones escolares que fueron aprobadas por la Monarquía y que fueron terminadas por la República en los años previos a la guerra; igualmente, son frecuentes los casos de escuelas cuyos expedientes se iniciaron este último período y que fueron construidas en la posguerra». Ante la pregunta de a quién adjudicar dichas obras y con qué criterio, opina este autor - contrariamente a Pilar García- que la paternidad debe atribuirse al sistema vigente en el momento de la finalización de la obra; y que ésta - continúa - «debe considerarse de facto cuando, al menos, se hubieran cubierto aguas, es decir, en el momento del pago del primer plazo de la subvención, en su caso». Es por aplicación de estos dos distintos criterios por lo que en la tabla anterior, y para un mismo año, mientras que para MPGS nunca habrá más escuelas construidas que programadas, para FJRM sí puede haberlas (por ejemplo en 1937, en que se terminaron más de las que se iniciaron). Una vez más, opinamos que los dos criterios debieran ser tenidos en cuenta. 
Del tercer capítulo — «Apuntes sobre la arquitectura escolar española de los años Veinte y Treinta»- me interesa especialmente, como estudioso de las construcciones escolares del período 1922-1937, la arquitectura producida al margen de la Oficina Técnica de Antonio Flórez, cuyas realizaciones han sido ya abordadas en numerosas publicaciones. En concreto me refiero a la labor de los arquitectos que trabajaron en colaboración con las Cajas de Ahorro y el Instituto Nacional de Previsión, insuficientemente estudiada hasta ahora. Lo aportado por la autora en este sentido, que es mucho tanto en textos como en imágenes, merece ser complementado desde diferentes enfoques en futuras investigaciones.

Sin embargo, es a mi juicio este capítulo, de los tres que integran el libro, el que entraña una menor novedad. Su objeto - la arquitectura escolar española de los años Veinte y Treinta- ha sido tratado ya por un buen número de autores. Además de los citados por la autora — quien, junto con sus propias observaciones, se basa fundamentalmente en Burgos Ruiz, Vázquez Astorga y Visedo Godínez-, traigo ahora a la memoria a Añón Abajas, ${ }^{17}$ Cabieces Ibarrondo, ${ }^{18}$ Guerrero, ${ }^{19}$ Mayol Amengual ${ }^{20}$ y más que me dejo en el tintero. Entre estos últimos también me incluyo, $\mathrm{y}$ he de reconocer que a uno le hubiera gustado ver contrastadas sus aportaciones con las de la autora, pues casi sobre todo ello hemos investigado, primero en la tesis doctoral ${ }^{21}$ y luego en los artículos y demás publicaciones que he ido desgranando. He de agradecerle las dos citas: la del artículo sobre el Grupo escolar «Gumersindo de Azcárate» de León y la del librito sobre la Escuela Normal de Zamora, que tuve el honor de coescribir con el profesor Hernández Díaz.

\footnotetext{
${ }_{17}$ Rosa-María Añón Abajas, La arquitectura de las escuelas primarias municipales de Sevilla hasta 1937 (Sevilla: Consejería de Obras Públicas y Transportes, 2005).

18 María Victoria Cabieces Ibarrondo, La arquitectura de los centros docentes en Cantabria en los siglos XIX y XX, Tesis doctoral (Santander: Universidad de Cantabria, 2016).

19 Salvador Guerrero López y otros, Antonio Flórez, arquitecto (1877-1941) (Madrid: Residencia de Estudiantes, 2002).

20 Jaume Mayol Amengual, L'arquitectura escolar de Guillem Forteza 1917-1943, Tesis doctoral (Barcelona: Universitat Politècnica de Catalunya, 2010).

${ }^{21}$ Francisco Javier Rodríguez Méndez, Arquitectura escolar en España (1857-1936). Madrid como paradigma, Tesis doctoral (Madrid: Universidad Politécnica de Madrid, 2004).
} 
Solo me queda desear que el libro de Pilar García Salmerón pase a engrosar la bibliografía de las publicaciones y tesis que en este campo están por venir, y que sea citada adecuadamente, pues a eso - ya que no a enriquecerse - es a lo que aspira todo investigador, y ella lo merece con creces.

\section{MARÍA DEL PILAR GARCÍA SALMERÓN}

Después de leer la reseña del profesor Francisco Javier Rodríguez Méndez sobre mi libro, Radiografía de las construcciones escolares públicas en España, 1922-1937, y aprovechando la oportunidad que me brinda el director de Historia y Memoria de la Educación, me gustaría comentar lo siguiente:

El libro se articula en dos partes diferenciadas; la primera incluye una descripción de las condiciones y circunstancias económicas, sociales, políticas y pedagógicas que favorecieron las construcciones escolares en aquellos años, especificándose los cambios más relevantes que permitieron desbloquear el alzado de nuevos centros. A continuación se analizan los resultados obtenidos sobre el número de aulas y edificios proyectados y/o acabados durante la etapa para concluir con una reflexión sobre la arquitectura escolar del momento, tanto la desarrollada por profesionales de la Oficina Técnica como por otros arquitectos independientes.

La segunda parte es un extenso Apéndice Documental, en que se incluyen unos listados, en los que aparecen, agrupados por provincias y ordenados cronológicamente, las referencias de todos los edificios escolares públicos cuya construcción se aprobó oficialmente durante la etapa, unas tablas en las que se resumen estos datos por anualidades y provincias, así como una gran cantidad de fotografías y planos.

Desde mi punto de vista, la novedad de esta exhaustiva investigación radica en varias cuestiones. Primero, el estudio se circunscribe a todo el territorio nacional, ofreciendo una panorámica general sobre el tema en un intervalo temporal que bien pudiera marcar la consolidación de la escuela primaria pública dentro del sistema educativo español, con la implicación decidida del Estado en la mejora de este nivel de enseñanza, que hasta ese momento había permanecido, casi en exclusiva, en manos 
de los municipios. El hecho de que a partir de los años veinte el Estado se involucre en la construcción de nuevas escuelas constituirá un paso decisivo que permitirá la paulatina mejora de la calidad de la enseñanza y, a largo plazo, la universalización de la escolaridad en España, así como modificar el tradicional y despectivo concepto de escuela pública. En segundo lugar, el libro desgrana detalladamente las circunstancias, los agentes y factores que facilitaron este hecho, que se presenta como un proceso a lo largo de toda la etapa, y no como un cambio súbito, fruto de una política educativa concreta asociada a un gobierno determinado. La asunción de los ideales regeneracionistas por los políticos y la sociedad, concretada tanto en la implantación del sistema organizativo graduado como en el aumento y mejora de calidad de la enseñanza pública, la creación de la Oficina Técnica para la Construcción de Escuelas, la disposición por el Estado de créditos extraordinarios para este cometido, así como la implicación de otras instituciones como el Instituto Nacional de Previsión y sus Cajas de Ahorro colaboradoras, crearán un caldo de cultivo que permitirá la emersión de cientos de nuevos edificios escolares por todo el paisaje español, siempre bajo la iniciativa de corporaciones municipales de todas las tendencias políticas. Desde mi punto de vista, desligar el incremento del número de escuelas alzadas de una corriente política determinada y asociarlo a otras circunstancias y agentes constituye uno de puntos más relevantes del libro. El volumen de documentación consultada para llegar a esta conclusión ha sido considerable, y me ha permitido descubrir la intrahistoria más desconocida ligada a la gestación y alumbramiento de una nueva escuela en distintas localidades. Tanto a través de las instancias que los alcaldes enviaban al Ministerio con la finalidad de justificar la necesidad de levantar una nueva escuela en su localidad, como a través de las crónicas que adornaban las inauguraciones de los nuevos centros, he podido tomar el pulso a la España más profunda, tal y como queda reflejado en algunas páginas del libro en las que se incluyen algunos de estos relatos.

Durante la etapa estudiada la construcción de escuelas siempre fue promovida por los municipios, que en la mayoría de los casos recibieron un auxilio económico por parte del Estado, y que a la hora de ejecutar las obras podían optar bien por acometerlas ellos mismos, bajo la supervisión de su arquitecto municipal, o bien encomendarlas al Estado, que a su vez, tras una subasta pública, las adjudicaría a un contratista, quien 
debía someterse a la dirección de un arquitecto de la Oficina Técnica para Construcción de Escuelas. A la primera fórmula se la denomina en el libro, construcción municipal, a la segunda, construcción tutelada por el Estado, si bien a continuación se aclara que en ambos casos el promotor de la construcción siempre fue el ayuntamiento respectivo. No hubo, pues, competencia alguna entre las administraciones local y central, sólo mayor o menor efectividad en el modo de acometer las obras. Tras consultar cientos de expedientes, parece que las obras tuteladas por el Estado tenían más posibilidades de finalizarse que las vigiladas por los ayuntamientos, si bien la tramitación administrativa de las primeras resultaba más lenta y cara que la segunda. Este punto resulta de capital importancia para entender las conclusiones finales de la investigación, ya que el ritmo y forma en la expansión de la red escolar pública durante estos años fueron marcados por los municipios, y secundados o acompasados por la acción del Estado, que se limitó a subvencionar aquellos proyectos que llegaron a sus manos, y que en ningún momento planificó a nivel nacional la ubicación de las nuevas escuelas. Salvo casos excepcionales, especificados en el libro, en los que el Estado decidió levantar algún centro escolar modélico en determinadas localidades, el resto de escuelas de nueva planta fueron promovidas por los municipios, entidades que debían proporcionar el solar y realizar una contribución económica, así como incoar el expediente que una vez tramitado y aprobado por el Ministerio de Instrucción Pública, aseguraría al municipio el respaldo económico estatal, aunque no garantizara que la escuela se construyera, pues el ayuntamiento debía encontrar la fórmula para hacer efectiva su aportación económica, que solía rondar el 25\% del presupuesto de obras. Determinar qué ayuntamientos lograron cumplir sus compromisos con el Estado para alzar la nueva escuela, a través de la consulta de los expedientes de construcción custodiados en el Archivo General de la Administración, ha sido una de las tareas más laboriosas.

En la investigación se cuantifica por provincias el número de aulas públicas levantadas cada año, así como los totales de escuelas proyectadas y/o construidas en las dos subetapas, llegando a la conclusión de que las provincias más activas durante los años veinte lo fueron también en los años treinta, probablemente porque los agentes que más influyeron a la hora de que un ayuntamiento se embarcara en el proceso de construcción de un edificio escolar, no fueron ni los políticos de un gobierno 
determinado, ni los altos funcionarios de la administración central, sino personas ligadas a las Juntas Provinciales de Enseñanza, los Inspectores de Educación, los consejeros de las Cajas de Ahorro o los gestores de las Diputaciones Provinciales, además, claro está, de los alcaldes y concejales de cada localidad.

Esta panorámica general ofrecida en el libro permite realizar comparaciones entre provincias, entre ciudades, entre etapas, así como contextualizar y valorar casos extraordinarios como el de la ciudad de Madrid durante la II República. Durante esta etapa, la capital fue la ciudad en la que más edificios y aulas se levantaron, edificios por otro lado de mayor calidad y con un número de dependencias anejas superior al de otros centros semejantes. Madrid fue la ciudad en la que más se invirtió durante la II República, el escaparate del régimen, en contraste con otras ciudades en las que no se construyó ni una sola aula, como Valencia, Lugo o Cádiz.

Creo que también conviene resaltar la contribución que la investigación ofrece sobre los proyectos atribuidos a arquitectos independientes o municipales, que diseñaron edificios escolares variopintos, sobre los planes de construcción locales diseñados por ayuntamientos de grandes ciudades y diputaciones provinciales y sobre el papel del Instituto Nacional de Previsión y sus Cajas de Ahorro en el asunto, así como la descripción de la construcción de las nuevas Escuelas Normales y sus Graduadas Anejas, asuntos quizá poco estudiados hasta ahora.

A mi modo de ver, otro de los puntos fuertes del libro reside en la profusión de fuentes primarias consultadas, que han cristalizado en un Apéndice Documental que representa la mitad de las páginas del mismo. Como ya se ha indicado, en él se incluyen las referencias de la $G a$ ceta de todos los proyectos aprobados oficialmente durante estos años, la localización archivística de los planos de muchos de ellos, más de cuatrocientas fotografías, inéditas la mayoría, más de cien planos, así como unos extensos listados, con miles de entradas, organizados por provincias, a través de los cuales puede observarse el desarrollo administrativo y de las obras de los cientos de escuelas cuya construcción se inició en estos años, y que pienso, pueden constituir un punto de apoyo para nuevas investigaciones, además de justificar las conclusiones del estudio. 
También quisiera referirme a la metodología adoptada en la confección del libro. Han sido más de mil las cajas consultadas en la sección del Ministerio de Educación del Archivo General de la Administración y en otros archivos de la geografía española con el fin de conseguir un aval casi irrefutable a las cifras presentadas. A través de esta documentación, que incluye desde el devenir administrativo de los expedientes abiertos en el Ministerio para tramitar los proyectos de construcción incoados por los ayuntamientos respectivos, hasta las minutas de los arquitectos y las certificaciones de obra, etc, se han obtenido las pistas y datos que han permitido estimar cuántos de los proyectos aprobados llegaron a franquear los obstáculos y barreras económicas hasta convertirse en un edificio escolar antes de 1937, fecha en la que se cierra la investigación, si bien en muchos casos se aportan referencias de edificios terminados a los pocos años de la conclusión de la Guerra.

Respecto a la disparidad de cifras, entre la contabilidad presentada en el libro y las que incluye el profesor Rodríguez Méndez, sin duda obedecen a la distinta metodología empleada, aunque la mía puede revisarse a través de los listados que presento, incluso rebatirse, confeccionando otros listados que avalen otras cifras, basadas en nuevas fuentes documentales. Dentro de este apartado, habría que comentar el punto referido a la contabilidad de aquellos centros y aulas con proyectos aprobados en 1936 y 1937, y cuyo alzado no se concluyó por diversos motivos, en especial por el estallido de la Guerra, pero mi trabajo termina en el año 1937 y por lo tanto no he realizado el seguimiento de estos centros. En todo caso, se deja claro que el número de proyectos aprobados oficialmente durante la II República fue mayor que el de los aprobados en la etapa anterior.

Por otro lado, acepto la observación del profesor Rodríguez Méndez sobre la posibilidad de contabilizar las dependencias anejas o complementarias a las aulas en los edificios escolares. Ciertamente, podría haberse hecho el recuento de esta manera, quizá habría respondido más exactamente a las cantidades invertidas, aunque yo opté por atenerme a la descripción del edificio escolar aparecida en la Gaceta, y consignar sólo las aulas ordinarias, si bien en los listados aparecen consignados los edificios que contaban con estas estancias, especificando su número y tipología, que no se han computado en ninguna de las subetapas consideradas. Quizá algún investigador se atreva a realizar este recuento en un futuro, 
incluso los listados incluidos en el libro le permitirán hacerlo con el mínimo esfuerzo. De pasada debo precisar que el número de dependencias complementarias, museo escolar, cantinas, bibliotecas, duchas, salas de trabajos manuales o labores, se fue reduciendo a medida que pasaban los años, ya que el esfuerzo inversor se fue decantando por conseguir la escolarización universal más que por lograr una enseñanza integral.

En cuanto a la propuesta del profesor Rodríguez Méndez de denominar aulas o edificios programados a los que contaban con la aprobación oficial de Estado, lo que les aseguraba el respaldo económico, en lugar de titularlos como aulas o edificios proyectados, como yo he considerado, entiendo que la palabra proyecto tiene para un arquitecto una primera connotación relativa al proyecto arquitectónico, a los planos y memorias que estos técnicos elaboran, pero el término proyecto también se refiere a cualquier acontecimiento programado para el futuro, a algo embrionario, en potencia, que puede o no desarrollarse en un futuro. Cuando en la investigación se habla de aulas o edificios proyectados me refiero a los que contaban con un proyecto arquitectónico redactado por el técnico correspondiente, incluido en un expediente administrativo aprobado por el Ministerio, en el que se fijaba una subvención del Estado, y que podía o no llegar a ejecutarse, dependiendo de si el ayuntamiento cumplía o no sus compromisos económicos con el gobierno central.

Por último, me gustaría resaltar como un valor singular, la novedad que esta obra representa dentro de la bibliografía sobre el tema, al haberse enfrentado a la cuestión con un extenso trabajo de campo realizado a través de varios años, incluyendo una contabilidad basada en fuentes primarias, así como el haber aclarado conceptos, confundidos a menudo por los estudiosos del tema, quienes asimilan y confunden términos como creación escolar con construcción escolar, o aulas con escuelas, o proyecto aprobado con edificio escolar construido, errores que han conducido a presentar como balance de las políticas educativas de estos años cifras desorbitadas que, según queda demostrado en el libro, ni se aproximan a lo acaecido realmente.

\section{ANTONIO VIÑAO FRAGO}

Ya desde sus inicios la recuperación académica de la educación republicana dio origen, entre otras polémicas, al debate acerca del alcance de 
la labor de construcción y creación de escuelas durante dicho período en relación con el precedente de la dictadura primorriverista y el posterior de la dictadura franquista. Los cálculos ofrecidos sobre el número de escuelas construidas o creadas desde 1931 a 1936 diferían según la ideología, enfoque o metodología seguida por quien los efectuaba. Como se indica en el texto introductorio de este debate, el artículo, publicado en 2013, de $M^{\mathrm{a}}$ del Pilar García Salmerón, redactado tras una investigación minuciosa, reabrió la polémica con el fin de rebatir, en este punto, las «inexactitudes descomunales» que a su juicio se vierten en el catálogo de la exposición La escuela de la II República organizada en 2007 por la Fundación de Investigaciones Educativas y Sindicales de la Federación de Enseñanza de Comisiones Obreras, ${ }^{22}$ así como de desmontar «el tópico que atribuye al advenimiento republicano el mérito del mayor incremento en las infraestructuras escolares de la historia contemporánea».23

Dejo a un lado el análisis que García Salmerón efectúa de los datos que ella misma aporta tal y como figuran en el texto introductorio de este «debate». Las 4.000 aulas construidas en los años veinte —es decir, a lo largo de diez años-, suponen una media de 400 aulas/año, mientras que las 3.000 aulas del período republicano — descartando la Guerra Civil, serían cinco años- ofrecen una media de 500 aulas/año, en un contexto económico, por cierto, radicalmente opuesto al de los años precedentes a la crisis de 1929. De un modo u otro, lo que las cifras muestran, en efecto, no es una «drástica mejora», sino la continuidad de una política de construcciones escolares propiciada, a nivel estatal, por la creación en 1920 de la Oficina Técnica para Construcción de Escuelas y reforzada durante la dictadura primorriverista gracias al papel clave desempeñado en dicha política por el Instituto Nacional de Previsión y las Cajas de Ahorro. ${ }^{24}$ Y, junto a ello, la importancia en dichos años de la iniciativa municipal, bien acometiendo directamente, con fondos propios, la construcción de escuelas, bien acogiéndose a las subvenciones y préstamos estatales, lo que explica, en buena parte, las sensibles

\footnotetext{
22 María del Pilar García Salmerón, «Radiografía de las construcciones escolares públicas en España (1920-1937): la imagen distorsionada de la II República»: Aportes XXVIII, no. 83 (2013): 21-52 (cita en p. 23).

${ }^{23}$ García Salmerón, «Radiografía de las construcciones escolares públicas en España (1920-1937)», 21.

${ }^{24}$ Instituto Nacional de Previsión, Fomento de construcción de escuelas. Instrucciones para la tramitación y concesión de préstamos a los ayuntamientos (Madrid 1924).
} 
diferencias, señaladas por García Salmerón, entre los municipios o provincias. En 1926 — todo un síntoma de la colaboración Estado-municipios- existía ya un grupo de arquitectos a nivel provincial o municipal -48 en total- especializados en la construcción de edificios escolares, que dirigen un escrito al gobierno instándole a que reconozca a nivel formal la existencia de algo así como unos servicios periféricos, a su cargo, de la mencionada Oficina Técnica. ${ }^{25}$ ¿Quiénes firman este escrito? Buena parte de los arquitectos que van a encargarse de redactar y dirigir las obras de construcción de escuelas en el período republicano. Una vez más, continuidad.

Dicho esto, lo que me interesa ahora no es tanto terciar en el debate ofreciendo mis propias cifras al respecto, cuanto señalar algunas cuestiones metodológicas con el doble fin de dar cuenta de las dificultades que reviste cualquier cálculo sobre el tema, y poner sobre aviso a quien pretenda efectuarlo.

En efecto, en estos cálculos habría que distinguir, a la hora de asignar la construcción a uno u otro año, entre: a) la iniciativa o propuesta; b) la inclusión de la obra en un programa de construcción de escuelas para unos años determinados; c) el encargo del proyecto de construcción, algo que solo puede o debe hacerse cuando hay financiación, pero que a veces se encarga mientras se tramita la misma; d) la redacción y aprobación del proyecto de obra; e) la contratación de la obra, que requiere la aprobación previa de su financiación y puede llevarse a cabo meses o años después del encargo del proyecto; f) su inicio; g) la terminación y recepción oficial de la obra, hecho que puede tener lugar varios meses o años después de la contratación o incluso del inicio: las escuelas inauguradas en 1931 y 1932 corresponden a proyectos, como es lógico, programados y comenzados en los dos, tres o incluso más años precedentes; y h) la puesta en funcionamiento de la escuela una vez dotada de material y haberse nombrado el profesorado.

Además, habría que tener en cuenta: a) si las unidades escolares construidas son para aulas de nueva creación (lo que exige que estén dotadas en plantilla) o de sustitución de otras en deficientes condiciones, que es como creo (hipótesis) que pasó con buena parte de las

${ }_{25}$ Los Arquitectos Escolares al servicio del Gobierno de Su Majestad (Madrid 1926). 
construidas o iniciadas en los años veinte y treinta; b) si, en las estadísticas o textos manejados, el término escuela se identifica con el de aula $\mathrm{o}$, por el contrario, se refiere a un edificio escolar con varias aulas; y c) que puede crearse una nueva aula, con dotación en plantilla, sin necesidad de que exista una construcción previa (locales habilitados, cedidos, alquilados, etc.). Lo relevante no sería, pues, tanto la construcción, aun siéndolo, como la dotación de una plaza de plantilla. Entiendo que cualquier valoración al respecto — sin desdeñar la importancia de sustituir aulas en malas condiciones por otras de nueva construcción - tendría que hacerse a partir de la evolución de la plantilla del magisterio primario algo, por otra parte, más fácil de determinar que lo relativo a la construcción de escuelas, dado que esta última implica un período que, como hemos visto, puede muy bien extenderse a lo largo de dos, tres o más años y adscribirse, por tanto, a alguno de ellos según el criterio o criterios adoptados.

Con independencia de todo ello, una cosa son los hechos, otra su interpretación — siempre contextual—y otra su valoración. En cuanto a los hechos, no es posible negar ni el fuerte incremento del presupuesto estatal dedicado a la educación primaria durante el primer bienio republicano, ${ }^{26}$ ni el de la plantilla de su profesorado - 28.924 plazas en 1923 , 36.680 en 1931 y 51.593 en 1935; es decir, un incremento de 964 maestros/año entre 1923 y 1931 y de 3.728 maestros/año entre 1931 y 1935 ${ }^{27}$, o de sus retribuciones, ni dejar se situar tales hechos en el contexto de una política de mayores exigencias en cuanto a la titulación de ingreso en las Escuelas Normales y la formación del magisterio primario. La valoración de tales hechos, y su calificación como mejora, progreso o avance, o como atraso, empobrecimiento o deterioro, en relación con períodos precedentes o posteriores, depende de las posiciones ideológicas o educativo-pedagógicas de quien escribe la historia, así como de su concepción del mundo y de la vida y del modelo de sociedad que se tiene en mente.

\footnotetext{
${ }^{26}$ Francisco Comín, Historia de la Hacienda Pública, II. España (1808-1895) (Barcelona: Crítica, 1996), 24-29-32, 38, 40, 42-43 y 224, y Hacienda y economía en la España contemporánea (1800-1936). Volumen II. La Hacienda transicional (1875-1935) (Madrid: Instituto de Estudios Fiscales, 1988), 636-638 y 1003-1004.

27 Las cifras proceden, en 1923, de la Estadística de Escuelas Nacionales de dicho año y, en 1931 y 1935, de los presupuestos generales del Estado.
} 
Desde una perspectiva más general, en relación con el proceso escolarizador básico, la cuestión se complica más todavía. Tras el debate sobre si el énfasis que se puso en la política de construcciones escolares durante la Segunda República fue inferior o superior al de la dictadura primorriverista, subyace una valoración positiva de la escolarización como avance o progreso que se transfiere al hecho de construir más o menos escuelas. Hoy, sin embargo, sabemos que la extensión y generalización de la escolarización básica se ha debido históricamente y ha estado ligada a factores tan diversos como el adoctrinamiento y proselitismo religioso, ideológico o político, la configuración del Estado moderno o del Estado-nación, la aculturación étnica o religiosa, a objetivos bélico-militares o económicos de índole general o particular, a la necesidad de proporcionar una formación cívica común, o a una educación considerada adecuada a la clases populares o trabajadoras conforme se ampliaba el derecho al voto o, simplemente, con el fin de desmontar la incipiente red escolar y cultural creada, al margen del Estado, por el movimiento obrero. En otras palabras, la escolarización, y con ella la construcción de escuelas, tiene muchas caras. Tantas como objetivos. Alguien —Carlos Lerena- las sintetizó en dos: reprimir y liberar. ${ }^{28}$ Reprime y libera en dosis diferentes según los objetivos, los contextos y los modos de escolarización, pero al mismo tiempo. Mejor dejar en este tema las valoraciones a un lado. $\mathrm{O}$, mejor dicho, tenerlas en cuenta, pero no en el sentido de equiparar sin más construcción de escuelas con nociones tan problemáticas como las de mejora o progreso.

Todo lo anterior no es óbice para reconocer la relevancia de la investigación llevada a cabo por María Pilar García Salmerón. Primero, por haber planteado una cuestión en la que, en efecto, se tiende más a las simplificaciones y los tópicos que a análisis rigurosos. Después, por su índole exhaustiva y meticulosa. Por último, entre otras razones, porque permite, con los datos aportados en el apéndice documental, efectuar análisis comparativos y diacrónicos entre unas regiones, provincias o localidades y otras. Calibrar los ritmos en el tiempo de la construcción de escuelas y captar dónde — distribución y desigualdades territoriales- y cuándo se puso el acento en unos u otros modelos de edificios

\footnotetext{
${ }_{28}$ Carlos Lerena, Reprimir y liberar. Crítica sociológica de la educación y de la cultura contemporáneas (Madrid: Akal, 1983).
} 
escolares. Un ejemplo que hemos analizado en un trabajo reciente ${ }^{29}$ en el caso madrileño donde, a diferencia de otros lugares, los desencuentros entre el Ministerio y el Ayuntamiento supusieron, al iniciarse la dictadura primorriverista, la paralización durante dos años del programa de construcción de escuelas aprobado en 1922. Un programa relanzado en 1925 y reforzado tras la proclamación de la Segunda República en 1931 con el fin de convertir la capital del Estado en el «escaparate» de la política educativa, en este ámbito, del régimen republicano. Pero, como acaba e indicarse, este sería un caso más entre otros más o menos singulares. No en todas las localidades y ayuntamientos se darían las mismas circunstancias o una evolución temporal similar. Captar y analizar dichas diferencias es una de las posibilidades que ofrece el libro de María Pilar García Salmerón. Otra, quizás la más relevante, determinar las continuidades y disimilitudes, en este punto, entre la dictadura primorriverista y la Segunda República.

\section{REFERENCIAS}

Alba Tercedor, Carlos. «La educación en la II República: un intento de socialización política», en Estudios sobre la Segunda República española, ed. Manuel Ramírez (Tecnos; Madrid 1975), 47-75.

Añón Abajas, Rosa-María. La arquitectura de las escuelas primarias municipales de Sevilla hasta 1937 (Sevilla: Consejería de Obras Públicas y Transportes, 2005).

Cabieces Ibarrondo, María Victoria. La arquitectura de los centros docentes en Cantabria en los siglos XIX y XX. Tesis doctoral (Santander: Universidad de Cantabria, 2016).

Cazarabet. El esfuerzo de la República en la construcción de nuevas escuelas en Aragón (1931-1937), a través de la Gazeta [consulta: 16-04-2016]. Disponible en: http://www.cazarabet.com/idea/escuelas/escuelasrepublica.htm.

Comín, Francisco. Hacienda y economía en la España contemporánea (18001936). Volumen II. La Hacienda transicional (1875-1935) (Madrid: Instituto de Estudios Fiscales, 1988).

Comín, Francisco. Historia de la Hacienda Pública, II. España (1808-1895) (Barcelona: Crítica, 1996).

\footnotetext{
29 Antonio Viñao, «Escuela graduada y medio urbano: construcciones, edificios y grupos escolares (1898/1936)» $\mathrm{y}$ "Graded schools in the urban settings: constructions, buildings and school complexes (1898/1936)», en Madrid, ciudad educadora 1898/1938. Memoria de la escuela pública (Madrid: Ayuntamiento de Madrid. Oficina de Derechos Humanos y Memoria, 2019), 168-265.
} 
Guerrero López, Salvador y otros, Antonio Flórez, arquitecto (1877-1941) (Madrid: Residencia de Estudiantes, 2002).

González Muñiz, Miguel Ángel. Problemas de la Segunda República (Ediciones Júcar; Madrid 1974), 327-407,

Fernández Soria, Juan Manuel. Educación y cultura en la guerra civil (España 1936-1939) (Nau Llibres: Valencia 1984).

García Salmerón, María del Pilar. La enseñanza en Cuenca durante la II República y la Guerra Civil Española. Tesis doctoral (Madrid: Universidad Nacional de Educación a Distancia, 1999).

García Salmerón, María del Pilar. Educación y República en Cuenca, 1931-1939 (Cuenca: Diputación Provincial de Cuenca, 2003).

García Salmerón, María del Pilar. «La política y las realizaciones educativas de la segunda República, a la luz de los estudios locales». Sarmiento. Anuario galego de historia da educación 8 (2004): 103-125.

García Salmerón, María del Pilar. «Radiografía de las construcciones escolares públicas en España (1920-1937): la imagen distorsionada de la II República». Aportes 28, no 83 (2013): 21-52.

García Salmerón, María del Pilar. Radiografía de las construcciones escolares públicas en España, 1922-1937 (Madrid: Ministerio de Educación y Formación Profesional, 2018).

Instituto Nacional de Previsión. Fomento de construcción de escuelas. Instrucciones para la tramitación y concesión de préstamos a los ayuntamientos (Madrid 1924).

Lerena, Carlos. Reprimir y liberar. Crítica sociológica de la educación y de la cultura contemporáneas (Madrid: Akal, 1983).

Los Arquitectos Escolares al servicio del Gobierno de Su Majestad (Madrid 1926).

Lozano, Claudio. La educación republicana, 1931-1939 (Universidad de Barcelona: Barcelona 1980),

Mayol Amengual, Jaume. L'arquitectura escolar de Guillem Forteza 1917-1943. Tesis doctoral (Barcelona: Universitat Politècnica de Catalunya, 2010).

Millán, Fernando. La revolución laica: de la Institución Libre de Enseñanza a la escuela de la República (Fernando Torres: Valencia 1983).

Molero Pintado, Antonio. La reforma educativa de la Segunda República española. Primer bienio (Santillana: Madrid 1977).

Pérez Galán, Mariano. La enseñanza en la Segunda República española (Edicusa: Madrid 1975).

Rodríguez Méndez, Francisco Javier. Arquitectura escolar en España (18571936). Madrid como paradigma, Tesis doctoral (Madrid: Universidad Politécnica de Madrid, 2004).

Rodríguez Méndez, Francisco Javier. «Pero, ¿cuántas escuelas construyó realmente la República? Parte primera: 1936-1939», en Actas de las VI Jornadas 
Científicas de la Sociedad Española para el Estudio del Patrimonio Histórico Educativo (Madrid, 2014), 567-575.

Safón, Ramón. La educación en la España revolucionaria (Las Ediciones de La Piqueta: Madrid. 1978).

Samaniego Boneu, Mercedes. La política educativa de la II República durante el bienio azañista (C.S.I.C.: Madrid 1977).

Vázquez, Matilde. «La reforma educativa en la zona republicana durante la guerra civil». Revista de Educación 240 (1975): 60-72.

Viñao, Antonio. «La "educación republicana" y algunos otros textos de Claudio Lozano", en La historia de la educación entre Europa y América: estudios en honor del profesor Claudio Lozano Seijas, dirs. Olegario Negrín Fajardo, Javier Vergara Ciordia y Conrado Vilanou Torrano, 103-114 (Madrid: Editorial Dykinson, 2018).

Viñao, Antonio. «Escuela graduada y medio urbano: construcciones, edificios y grupos escolares (1898/1936)» y «Graded schools in the urban settings: constructions, buildings and school complexes (1898/1936)». En Madrid, ciudad educadora 1898/1938. Memoria de la escuela pública, 168-265 (Madrid: Ayuntamiento de Madrid. Oficina de Derechos Humanos y Memoria, 2019). 